\title{
Escritura del anteproyecto en posgrado: experiencia de estudiantes de maestría en Salud*
}

\author{
Writing of the \\ Preliminary Research \\ Project in Graduate \\ Level: An Experience of \\ Students of a Master's \\ Degree in Health
}

\author{
Escritura do anteprojeto \\ em pós-graduação: \\ Experiência de estudantes \\ do mestrado em saúde
}

Laura Sofía Gómez Palomino** https://orcid.org/0000-0002-8479-8536

Paula Alejandra Peña Jaramillo*** https://orcid.org/0000-0003-1866-4086

Karina Alejandra Arenas-Hernández *** https://orcid.org/0000-0002-9378-8356
Para citar este artículo

Gómez-Palomino, L.S., Peña-Jaramillo, P.A. y Arena-Hernández, K.A. (2022). Escritura del anteproyecto en posgrado: experiencia de estudiantes de maestría en Salud. Folios, (55). https://doi.org/10.17227/folios.55-12477
* La información de este artículo se deriva del proyecto de investigación "Las prácticas pedagógicas para apoyar la escritura de la tesis en las maestrías en Salud Pública y Epidemiología de la Universidad del Valle", financiado por la convocatoria interna 2016-1 de la Vicerrectoría de Investigaciones de la Universidad del Valle.

** Fonoaudióloga. Universidad del Valle.

Correo: sofia.gomez@correounivalle.edu.co

*** Fonoaudióloga. Universidad del Valle.

Correo: paula.a.pena@correounivalle.edu.co

${ }^{* * * *}$ Fonoaudióloga, magíster en Lingüística y Español. Universidad del Valle.

Correo: karina.arenas@correounivalle.edu.co
Artículo recibido $15 \cdot 09 \cdot 2020$

Artículo aprobado $02 \cdot 06 \cdot 2021$ 


\section{Resumen}

Este artículo presenta una investigación cuyo objetivo fue caracterizar el proceso de escritura del anteproyecto de tres estudiantes de maestría en Salud, en términos de sus expectativas y conocimientos previos, sus avances en la escritura, la valoración que hicieron de los apoyos pedagógicos recibidos y los aspectos relacionados con su experiencia de escritura. La investigación se hizo en un curso de Metodología de la Investigación en el que hubo acompañamiento explícito en escritura; tuvo un enfoque cualitativo, un tipo de estudio descriptivo y se desarrolló a través de entrevistas estructuradas y análisis de contenido. Los principales hallazgos muestran que los estudiantes consideran que los apoyos explícitos en escritura fueron fundamentales para la elaboración del anteproyecto y los relacionan positivamente con los avances que tuvieron en la escritura. Sin embargo, se encontró que las emociones que se derivan del proceso de la escritura y los factores que influyen en este son principalmente negativos.

\section{Palabras clave}

escritura; proyecto de investigación; estudiante de posgrado; salud

\section{Abstract}

The present article exposes research whose main objective was to characterize the writing process of the preliminary research project of three master's students, in terms of their expectations and previous knowledge, the advances they had in the writing, the evaluation they made of the pedagogical supports received and the aspects related to their writing experience. The research had a qualitative approach and a type of descriptive study and was developed, mainly, through structured interviews. The main findings show that students consider explicit support for writing is necessary for the preparation process of the preliminary project and relate them positively to the advances they had in writing. On the other hand, it was found that the emotions derived from the writing process and the factors that influence it are mainly negative.

\section{Keywords}

writing; research projects; postgraduate education; health

\section{Resumo}

Este artigo apresenta uma pesquisa cujo objetivo foi caracterizar o processo de escrita do anteprojeto de três alunos de mestrado em saúde, em termos de expectativas e conhecimentos prévios, seu progresso na escrita, a avaliação que fizeram dos apoios pedagógicos recebidos e os aspectos relacionados à sua experiência de escrita. A pesquisa foi realizada em um curso de Metodologia de investigação, em que havia acompanhamento explícito na escrita; teve uma abordagem qualitativa, um tipo de estudo descritivo, e foi desenvolvido por meio de entrevistas estruturadas e análise de conteúdo. As principais conclusões mostram que os alunos consideraram que os apoios explícitos na escrita eram fundamentais para a preparação do anteprojeto e os relacionaram positivamente com os avanços que tiveram na escrita. Por outro lado, verificou-se que as emoções decorrentes do processo de escrita e os fatores que o influenciam são principalmente negativos.

\section{Palavras chave}

escrita; projeto de pesquisa; aluno de pós-graduação; saúde 


\section{Introducción}

La escritura en el posgrado requiere un nivel mayor de exigencia respecto al pregrado, por lo que la elaboración de la tesis de investigación se presenta como un desafío y, en muchas ocasiones, se convierte en un obstáculo para obtener el título. Al respecto, Carlino menciona que: "Hacer la tesis como parte de un posgrado que requiere una producción escrita original es la fase más ardua del trabajo, la que más demora y la que suele poner los escollos más difíciles de remontar" (2005, p. 2).

La revisión de antecedentes investigativos sobre escritura en posgrado muestra las siguientes tendencias: las prácticas de los docentes para guiar la escritura, como por ejemplo, las tutorías en diferentes modalidades como la "tutoría de pares" y la "tutoría grupal" (Mostacero, 2014) o la exploración del género textual, escritura de diario de tesis y revisión entre pares (Carlino, 2009); y las diferentes formas o estrategias para ocuparse de la enseñanza de la escritura en universidades de varios países, como Australia (Carlino, 2002), Canadá, Argentina, Estados Unidos (Carlino, 2009) y Colombia (Uribe y Camargo, 2011).

Además, se han estudiado, por una parte, factores que influyen positivamente en la escritura de los estudiantes, tales como leer y escribir para el desa- rrollo de la tesis (Ochoa, 2009) y el uso de estrategias metacognitivas (Chirinos, 2012), y por otra, aquellos factores que la obstaculizan, por ejemplo, la dificultad para escribir teniendo en cuenta la audiencia a la que se dirige el texto, el desaprovechamiento del potencial epistémico de la escritura, la propensión a revisar los textos solo en forma lineal y centrándose en aspectos locales y poco sustantivos, y la postergación del momento de empezar a escribir (Carlino, 2005). Chois y Jaramillo (2016) publicaron un estado del arte sobre la escritura en posgrado, en el que analizaron 39 documentos e identificaron los ejes temáticos más investigados en este campo y los países en los que se han adelantado los trabajos. Por su parte, Vargas Franco (2016) realizó un estudio de caso desde la perspectiva de un estudiante de posgrado acerca de la escritura de su trabajo de grado; este último es el estudio más similar a la presente investigación, pero en otra área del conocimiento.

La alfabetización académica propone que la lectura y la escritura se asuman como procesos que favorezcan la construcción de conocimiento en cada disciplina, no como prácticas aisladas en materias especializadas, lo que implica abordarlas en el seno de las asignaturas del currículo (Carlino, 2013). Precisamente, el contexto de la investigación en la que se enmarca este artículo se sitúa en la Maestría 
en Salud Pública de la Universidad del Valle, en el curso Metodología de la Investigación I, en el que los estudiantes contaron con un módulo de escritura, articulado a las temáticas propias del seminario. La investigación tuvo el propósito de caracterizar el proceso de escritura del anteproyecto de grado de tres estudiantes de dicha maestría. Los objetivos específicos estuvieron orientados a indagar sobre los conocimientos previos y expectativas que tenían de su proceso de escritura, describir los cambios que se evidencian en tres versiones del anteproyecto, conocer la valoración que hicieron los estudiantes de los apoyos pedagógicos recibidos para la escritura e identificar factores emocionales, personales, laborales o académicos que influyeron en el proceso de escritura del texto mencionado.

El interés en el estudio surgió del contexto particular del curso mencionado, ya que hay orientación explícita de la escritura de la tesis, asunto poco frecuente en las experiencias de investigación reportadas en posgrado y que representa un aporte a las investigaciones interesadas en evidenciar los efectos de definir estrategias de acompañamiento para la escritura de los estudiantes de este nivel.

\section{Metodología}

La investigación se desarrolló desde un enfoque cualitativo y un tipo de estudio descriptivo, ya que se buscó tener en cuenta la perspectiva de los participantes en su ambiente natural y, en relación con el contexto, profundizar en sus experiencias, perspectivas y opiniones (Hernández Sampieri et al., 2010). El objetivo fue caracterizar el proceso de escritura que llevaron a cabo tres estudiantes de la Maestría en Salud Pública de la Universidad del Valle para construir su anteproyecto de grado, desde la perspectiva de los conocimientos y expectativas previas que tenían sobre la escritura de este texto, los cambios que se evidencian en distintas versiones del anteproyecto, las valoraciones que hacen de los apoyos recibidos durante la asignatura por parte de las docentes y los factores emocionales, personales, laborales o académicos que influyeron en el proceso de escritura del anteproyecto.
Este estudio se derivó del proyecto "Las prácticas pedagógicas para apoyar la escritura de la tesis en las maestrías en Salud Pública y Epidemiología de la Universidad del Valle", dirigido por docentes de las Escuelas de Salud Pública y de Rehabilitación Humana (Chois-Lenis et al., 2020). Para el proyecto, se seleccionó una muestra de tres estudiantes de Salud Pública que estuvieran cursando la asignatura Metodología de la investigación I, de acuerdo con sus notas finales, que los ubicaron en niveles de desempeño bajo, medio y alto. Esta misma muestra se tuvo en cuenta para la investigación reportada en este artículo.

Se usaron entrevistas estructuradas para los objetivos relacionados con la identificación de los conocimientos y las expectativas de los estudiantes respecto a su proceso de escritura del anteproyecto; así como las valoraciones que hicieron de los apoyos brindados por los docentes, y los factores emocionales, personales, laborales o académicos que influyeron en su proceso de escritura. Para la descripción de los cambios que se evidenciaron en los anteproyectos de grado, se usó la técnica de análisis de contenido de tres versiones de estos, con el fin de identificar los tipos de cambios que hubo en los textos, teniendo en cuenta las indicaciones de las docentes encargadas de la asignatura y una guía de contenidos basada en lo dispuesto por el Departamento Administrativo de Ciencia, Tecnología e Innovación (Colciencias), usada por ellas.

En la tabla 1, se presentan las categorías de análisis usadas para procesar la información recolectada. 
Tabla 1. Categorías de análisis

\begin{tabular}{|c|c|c|}
\hline $\begin{array}{l}\text { Información } \\
\text { recolectada }\end{array}$ & Categoria & $\begin{array}{l}\text { Descripción de } \\
\text { la categoria }\end{array}$ \\
\hline \multirow{5}{*}{$\begin{array}{l}\text { Conocimientos y expectativas } \\
\text { previas de los estudiantes } \\
\text { sobre su proceso de escritura }\end{array}$} & $\begin{array}{l}\text { Concepciones del proceso de } \\
\text { escritura }\end{array}$ & $\begin{array}{l}\text { Percepción y descripción sobre en qué consiste el proceso } \\
\text { de escritura de un anteproyecto de grado. }\end{array}$ \\
\hline & Demandas de la escritura & Percepción sobre las exigencias de la escritura académica. \\
\hline & Etapas de la escritura & $\begin{array}{l}\text { Percepción sobre las etapas que requiere el proceso de } \\
\text { escritura. }\end{array}$ \\
\hline & Condiciones de la escritura & $\begin{array}{l}\text { Percepción sobre los requisitos o particularidades con los } \\
\text { que se debe abordar el proceso de escritura. }\end{array}$ \\
\hline & Apoyos pedagógicos & $\begin{array}{l}\text { Apoyos que los estudiantes esperan recibir de los docentes } \\
\text { para la escritura del anteproyecto. }\end{array}$ \\
\hline \multirow{3}{*}{ Contenidos del anteproyecto } & Planteamiento del problema & $\begin{array}{l}\text { Apartado en el que se establece formalmente el objeto de } \\
\text { estudio mediante preguntas que orientan los resultados } \\
\text { que se van a obtener. }\end{array}$ \\
\hline & Objetivos & $\begin{array}{l}\text { Propósitos con los que se realiza la investigación, que de- } \\
\text { ben guardar relación con la pregunta y el título de esta. }\end{array}$ \\
\hline & Metodología & $\begin{array}{l}\text { Apartado que explicita la forma en la que se desarrollará la } \\
\text { investigación de acuerdo con los objetivos. }\end{array}$ \\
\hline \multirow[t]{2}{*}{ Valoración de los apoyos } & $\begin{array}{l}\text { Apoyos pedagógicos recibidos du- } \\
\text { rante la asignatura para la } \\
\text { escritura del anteproyecto }\end{array}$ & $\begin{array}{l}\text { Mención de las diferentes ayudas o contribuciones que hi- } \\
\text { cieron los docentes en la asignatura para apoyar la escritu- } \\
\text { ra del anteproyecto. }\end{array}$ \\
\hline & Valoración de apoyos recibidos & $\begin{array}{l}\text { Percepción de los estudiantes sobre la utilidad de los apo- } \\
\text { yos recibidos. }\end{array}$ \\
\hline \multirow{2}{*}{$\begin{array}{l}\text { Factores que influyeron en el } \\
\text { proceso de escritura }\end{array}$} & $\begin{array}{l}\text { Factores que obstaculizan o } \\
\text { facilitan el proceso de escritura }\end{array}$ & $\begin{array}{l}\text { Aspectos laborales, académicos, personales o emociona- } \\
\text { les que influyen en el proceso de escritura. }\end{array}$ \\
\hline & $\begin{array}{l}\text { Emociones y sentimientos durante } \\
\text { el proceso de escritura }\end{array}$ & $\begin{array}{l}\text { Emociones y sentimientos positivos o negativos que se } \\
\text { identificaron durante el proceso de escritura. }\end{array}$ \\
\hline
\end{tabular}

Fuente: elaboración propia

\section{Resultados}

\section{Conocimientos y expectativas previas de los estudiantes}

A continuación, se describe el análisis de las entrevistas iniciales realizadas a los participantes de la investigación acerca de los conocimientos y expectativas sobre el proceso de escritura del anteproyecto. La información se organiza de acuerdo con las respuestas y particularidades de cada caso, y se cita lo referido por cada uno.

\section{Proceso de escritura}

El proceso de escritura fue descrito por los tres estudiantes como:

Un proceso largo. ¿Por qué? Requiere mayor rigor a nivel de redacción, debe ser claro y con ideas puntuales. $Y$ actualmente no se cuenta con estrategias de apoyo en el proceso de producción textual. (Estudiante 1)

Creo que el proceso de escribir un proyecto de investigación es dispendioso, en la medida en que a partir de la pertinencia de una temática de interés propio se deben desplegar una serie de pasos (Estudiante 2) 
a manera personal creo que puede resultar tedioso y estresante, no tengo ninguna experiencia en escritura de proyectos de investigación. Por lo tanto, es todo un reto para mí, es un proceso de aprendizaje. (Estudiante 3)

Estas concepciones muestran que los participantes ubican la escritura en el nivel de posgrado como un proceso de rigor, complejidad y profundidad que requiere de argumentación y sustentación, tal como lo plantea Castelló (2009a), quien los describe como "un proceso largo y complejo que requiere de una intensa actividad cognitiva que exige [...] buscar información, seleccionar [...] lo más relevante, ordenar y organizar esta información en función de los propios objetivos" (p. 122).

Por su parte, Arnoux et al. (2004) plantean que la escritura en posgrado exige un nivel mayor de conceptualización, integración de conocimientos y trabajo autónomo. Lo anterior provoca que el proceso a su vez implique otros factores, como las estrategias de escritura o apoyos que se brinden por parte de los docentes y la experiencia en escritura para tener mayor herramientas y conocimientos que faciliten el proceso (Espino Datsira, 2015), ya que estos desempeñan un papel fundamental para dar respuesta a las altas exigencias tanto cognitivas como discursivas en el proceso de escritura de un proyecto de investigación. Además, los estudiantes identifican como factor importante el interés como sentido inicial de la escritura y el estrés como un sentimiento que se deriva del proceso, lo cual es uno de los elementos que influyen en la eficacia del proceso de composición del texto (Flower y Hayes, 1996), pues hacen parte de las actitudes, creencias, motivaciones y otros factores afectivos.

\section{Demandas de escritura}

Respecto a las percepciones sobre lo que requiere o exige el proceso de composición, los estudiantes expresaron lo siguiente:

lectura constante, actividades de producción textual. (Estudiante 1)

creo que escribir mi proyecto de investigación me demandará, en primera instancia, máxima dedicación a la búsqueda de información en un primer momento y posteriormente una dedicación a la lectura juiciosa y estricta de los textos para posteriormente organizarlos de una forma coherente y lógica que permita al interlocutor un acercamiento a lo que se desea investigar en todos los apartes que contiene. Todo lo anterior está supeditado a unos tiempos de entrega y la valoración que haga el tutor a partir de la lectura realizada, lo que muy seguramente implicará el ejercicio de reescribir el texto. (Estudiante 2)

tener una buena búsqueda bibliográfica que me permita informarme acerca de lo que se sabe del tema que quiero investigar, la elaboración del estado del arte, así como establecer la metodología correcta para llevar a cabo el proyecto. Una vez terminado esto, la discusión de los resultados y sus conclusiones, para ver qué utilidad me da toda la escritura del proyecto. (Estudiante 3)

De lo anterior se puede resaltar que los estudiantes identifican elementos fundamentales para la escritura de textos académicos como los planteados por Castelló (2009b), dentro de los que se encuentran la búsqueda, selección, organización y revisión de información, además de planificación y control, que también son planteados como estrategias de lectura y escritura por autores como Zanotto et al. (2011) y Castelló et al. (2009). Dichos elementos se ubican de manera constante durante el proceso de producción textual y son de gran importancia ya que permiten que el escritor organice conscientemente su texto para responder a los objetivos iniciales y a las exigencias de la escritura. Además, los estudiantes consideran relevantes las demandas de tipo personal, como el tiempo y la dedicación, que se constituyen como factores que pueden influir en la eficacia del proceso de escritura (Flower y Hayes, 1996).

\section{Etapas de la escritura}

Los estudiantes reconocen diferentes etapas en el proceso de escritura. Las respuestas coinciden en que este implica la estructuración de un plan de escritura (lluvia de ideas, tablas, listas, etc.), escritura de acuerdo con los parámetros establecidos para 
el apartado, citación de fuentes y análisis de datos, con lo que se puede deducir que los estudiantes comprenden los pasos de escritura como apartados del texto que deben desarrollar o como reglas de escritura. Lo anterior lo expresan así:

Primero palabras claves, una lluvia de ideas, buscar coherencia y cohesividad en la estructura del texto. (Estudiante 1)

Con base en los resultados encontrados según la metodología que uno emplee, el análisis de datos. La elaboración de la discusión del proyecto debo hacerla con base en mis resultados y los de otros proyectos, citándolos haciendo comparaciones, sugerencias, etc. (Estudiante 3)

De las respuestas obtenidas, llama la atención la expresada por uno de los estudiantes, quien enumera y formula una lista de seis pasos de escritura:

1. Elaborar una tabla de contenido de las temáticas a desarrollar y los subtítulos para guiar la escritura.

2. Tener a la mano las citas que he escogido de las lecturas realizadas.

3. Escribir de acuerdo a la tabla de contenido propuesta respetando las condiciones de extensión propuestas y la normativa de referenciación.

4. Leer lo escrito y revisar algunas dificultades que tenga el mismo tanto en el contenido como en la forma, como también ideas que puedan ser confusas o estén repetidas.

5. Reescribir o mejorar el texto escrito de acuerdo a las fallas detectadas.

6. Realizar una última lectura y hacer ajustes finales. (Estudiante 2)

Los pasos mencionados se relacionan de manera coherente con los procesos y subprocesos descritos por Flower y Hayes (1996) ya que se corresponden con la planificación, la redacción, el examen y el control de la escritura. Sin embargo, de acuerdo con los autores, este proceso no se da de manera lineal y consecutiva en los contextos reales de escritura, como lo plantea el estudiante, sino de manera flexible durante todo el proceso de composición. Llama la atención que, por lo menos en lo relacionado con el conocimiento declarativo, los tres estudiantes parecen asumir comportamientos estratégicos en el proceso de escritura.

\section{Condiciones de la escritura}

Las respuestas sobre las condiciones necesarias para la escritura del anteproyecto que dieron los estudiantes fueron:

Se necesita primordialmente tiempo, disposición, dedicación. También es necesario contar con un apoyo para optimizar el proceso de redacción, podría ser un curso corto de producción textual. (Estudiante 1)

Considero que para el ejercicio de escribir el proyecto necesito dedicar un tiempo de calidad a dicho ejercicio lo cual implica menos horas de sueño y modificaciones a la demanda laboral actual. (Estudiante 2)

¡Tiempo! Pero más que tiempo, el buen uso de este, tener disciplina para cumplir planes de trabajo establecidos. La escritura de un proyecto es algo que uno sabe que requiere sacrificios, pero muchas veces no se es consciente de cuánto realmente cuesta esta producción intelectual. (Estudiante 3)

Lo anterior resalta lo mencionado por Castelló (2009a) sobre la escritura como un proceso "flexible, dinámico y diverso en el que cada escenario dibuja una trama de condiciones particulares y sugiere un modo de proceder diferente" (p. 50). Es decir que no hay un entorno ideal de escritura, ya que cada persona se enfrenta a su propio proceso de manera individual y diferente, por lo que no hay un estándar que condicione la escritura. Por otro lado, las respuestas obtenidas demuestran que los estudiantes coinciden en otorgarles un valor importante a factores como el tiempo, los apoyos y la disciplina durante el proceso. 


\section{Apoyos pedagógicos}

Respecto a los apoyos pedagógicos que esperaban recibir, los estudiantes coinciden en que los docentes debían intervenir

haciendo retroalimentación y brindando técnicas o estrategias para realizar un mejor proceso de redacción. (Estudiante 1)

realizando correcciones a los textos escritos en todo sentido; forma, contenido, suficiencia explicativa y conceptual. (Estudiante 2)

asesorías, revisión y corrección de avances, etc. (Estudiante 3)

Por estas razones, los estudiantes hacen referencia a la necesidad de brindar apoyos durante el proceso de escritura, lo cual se relaciona con lo que plantean Arnoux et al. (2004), quienes mencionan que "debido a las generalizadas dificultades de los tesistas, y a la escasa proporción que logra finalizar sus tesis, es preciso que los posgrados ofrezcan espacios continuados y diversificados para ayudar a hacer frente a los obstáculos descubiertos" (p. 14), y además se le otorga un valor importante al docente como apoyo fundamental, ya que la posibilidad que tiene el estudiante de aprender del asesor y revisor, y de sus observaciones y correcciones se considera como un factor favorecedor del proceso de escritura (Torres y Guadalupe, 2012).
En el contexto del curso estudiado, se resalta la oportunidad que tuvieron los estudiantes de contar con la orientación de dos docentes: una de ellas especialista en el área de conocimiento particular y otra experta en escritura académica, lo que refleja una característica poco común en los contextos de los posgrados: colaboración entre docentes para abordar asuntos de escritura académica de manera situada. Los apoyos comunes que brindaron estuvieron relacionados con lectura de textos de ejemplo del género que los estudiantes debían producir, formulación de indicaciones escritas para la elaboración del anteproyecto, comentarios escritos u orales en las versiones de los textos producidos por los estudiantes y el uso de la estrategia de revisión entre pares (Chois-Lenis et al., 2020).

\section{Análisis de las versiones de escritura del anteproyecto de grado de los estudiantes}

Teniendo en cuenta las orientaciones y exigencias de la docente especialista en el área de la asignatura, los contenidos que propone Colciencias en sus lineamientos para la escritura de la propuesta de investigación (usados por la docente) y el syllabus de la asignatura, se construyó la tabla 2 para el análisis de la estructura y los apartados incluidos en la propuesta de investigación de los maestrantes. 
Tabla 2. Elementos de análisis de la estructura y apartados del anteproyecto

Título

Planteamiento del problema de investigación

- Problema de salud pública

- Magnitud del problema de investigación

- Pregunta de investigación

- Antecedentes

\section{Objetivos}

Inician con un verbo en infinitivo; indican el tiempo, el lugar y los sujetos; evidencian la aproximación teórica; su redacción es comprensible.

- General: cumpliendo el objetivo general, se consigue responder a la pregunta de investigación.

- Específicos: los objetivos específicos dan claridad sobre la perspectiva teórica.

\section{Metodología}

- $\quad$ Tipo de estudio

- Área de estudio

- Población y muestra

- Variables

- Metodología

- Recolección de información y análisis

Fuente: elaboración propia

De acuerdo con el syllabus de la asignatura, las entregas de avances del anteproyecto estuvieron distribuidas de la siguiente manera:

- Primera entrega: planteamiento del problema de investigación.

- Segunda entrega: planteamiento del problema de investigación, objetivos y enfoque metodológico.

- Entrega final: versión completa del anteproyecto. Por cuestiones de tiempo, esta entrega fue modificada por la docente y se determinó que se entregarían los apartados completos de planteamiento, objetivos y metodología. El anteproyecto final se entregó en el semestre siguiente, periodo fuera del alcance del presente análisis.

A continuación, se presenta una descripción de cada una de las entregas de los estudiantes incluidos en la investigación. Posteriormente, se hace un análisis de las particularidades de sus avances y un balance de sus procesos de escritura, que permite identificar cuáles fueron los aportes de los apoyos con los que contaron los estudiantes.

\section{Estudiante 1}

Primera entrega

Identificamos que el maestrante entregó una primera versión correspondiente al planteamiento del problema de investigación, que cumple con la extensión exigida (máximo tres hojas), lo que concuerda con lo estipulado en el syllabus del curso y lo solicitado por la docente. Se observa que el apartado incluye cinco contenidos: magnitud del problema de salud pública, hallazgos e investigaciones sobre este, problema de investigación, brecha del conocimiento y pregunta de investigación. No se incluyen aspectos relacionados con la manera en que se va a responder a la pregunta y para qué serán utilizados los resultados de la investigación; sin embargo, esto no afecta la claridad ni la estructura del texto realizado. La propuesta del estudiante se enmarca en un macroproyecto de investigación realizado en una institución de educación superior, lo que permite evidenciar que hay una temática bien definida que va acorde con la guía de la docente, una claridad conceptual de los aspectos que se ven involucrados 
en la investigación y precisión de la pregunta de investigación.

\section{Segunda entrega}

En la segunda versión se identifica un documento más estructurado con portada, contraportada, división y numeración de los apartados incluidos, y bibliografía. Además de esto, ya se propone un título que explicita el tema de investigación con la población elegida y también se expone el director del proyecto. El documento cumple con dos de los apartados solicitados según el syllabus: planteamiento del problema, modificado según las sugerencias de la docente en la primera versión, y objetivos general y específicos; no se presenta el enfoque metodológico. Respecto al objetivo general, este es medible y está formulado con un verbo en infinitivo, plantea lo que se quiere hacer en la investigación y tiene coherencia con la pregunta de investigación y con el título. Con base en este, el estudiante plantea tres objetivos específicos que, de igual manera, tienen coherencia tanto con el objetivo general como con la pregunta de investigación, son formulados con verbos en infinitivo, son mutuamente excluyentes y dan cumplimiento de forma completa al objetivo. El estudiante se mantiene en la misma línea investigativa articulada al proyecto de la institución de educación superior.

\section{Tercera entrega}

El estudiante incluye en su entrega final los siguientes apartados: planteamiento del problema de investigación; objetivo general; objetivos específicos; enfoque metodológico, que contiene diseño, variables de resultado, variables de exposición, muestreo y análisis; y, por último, presenta las referencias bibliográficas. No se observan dentro del apartado de metodología los puntos de área de estudio, recolección de información y consideraciones éticas, exigidos en la guía. En cuanto a las variables, estas son organizadas en tablas que incluyen: categoría de análisis, variable, definición operacional e instrumento, lo que proporciona una amplia información, pero no se explicita el tipo de variable (independiente, dependiente, de exposición, etc.) ni los momentos de medición durante la investigación. En cuanto a la población y la muestra, la información proporcionada no es completa, ya que no presenta la descripción de las características generales de la población con la que se realizará el estudio, ni los criterios de inclusión y exclusión para la investigación. Tampoco se explican los procesos que se llevarán a cabo para la recolección de la información. Como último apartado del anteproyecto, el estudiante hace explícitos los tipos de análisis de datos que se realizarán (univariado, bivariado, multivariado) de manera muy general, por lo que el apartado tiene una extensión muy corta y no se presenta al lector una información suficiente para la comprensión total de lo que pretende realizar.

\section{Estudiante 2}

Este estudiante únicamente realizó dos entregas de anteproyecto para la asignatura.

\section{Primera entrega}

La primera entrega del estudiante incluye el planteamiento del problema, siguiendo lo estipulado en el syllabus, y los objetivos general y específicos. En este documento se identifican elementos como el encabezado con el nombre de la universidad, la maestría y la asignatura, y el nombre del autor del documento; organización mediante títulos y subtítulos; numeración de páginas, y el uso de subíndices y notas al pie de página para citas y referencias bibliográficas. Respecto al planteamiento del problema, este incluye el problema de investigación, la brecha del conocimiento y la pregunta de investigación, pero no se explicita información sobre la magnitud del problema a nivel mundial, nacional o regional, los hallazgos sobre la problemática en otras investigaciones, ni tampoco cómo se va a responder la pregunta planteada y para qué se van a usar los resultados de la investigación. De igual manera, este apartado excede las tres páginas permitidas, ya que las referencias bibliográficas se ubican en el pie de página. Respecto a los objetivos, se identifica que el general cumple con las especificaciones de la guía ya que es medible, está formulado 
con un verbo en infinitivo, plantea lo que se quiere hacer en la investigación y tiene coherencia con la pregunta de investigación. Del objetivo general se derivan tres específicos que tienen coherencia con la pregunta de investigación, son formulados con verbo en infinitivo, son mutuamente excluyentes y dan cumplimiento de forma completa al general.

Segunda entrega (final)

El documento de esta entrega se observa más estructurado que el anterior, incluye portada y contraportada, numeración de páginas, título de la investigación, nombre del autor y el director elegido, nombre de la universidad y nombre de la maestría. Como contenidos, están el planteamiento del problema de investigación, los objetivos, la metodología y la bibliografía. El planteamiento se observa mejor organizado, debido a que se añade más información acerca del problema de investigación, una conclusión y cambios en la pregunta de investigación que la hacen más precisa en cuanto a la población que se va a abordar. Estos cambios influyen también en la precisión de los objetivos, de tal forma que se evidencia coherencia entre los apartados. Respecto a la metodología, el estudiante ubica tipo de estudio, diseño, instrumentos de recolección de la información y categorías de análisis, pero deja por fuera puntos solicitados en la guía de escritura, como área de estudio, población y muestra, variables, plan de análisis y consideraciones éticas.

Respecto al tipo de estudio y el diseño, el estudiante únicamente presenta el nombre y una breve descripción de estos; no se evidencia un panorama general del estudio, información acerca de la razón de la elección del diseño, las variables de interés para el estudio, los retos que representa usar el diseño elegido y cómo los afrontará, por lo que este ítem no cumple con el mínimo de páginas referido en la guía (una página).

Igualmente, para los instrumentos de recolección de la información y categorías de análisis únicamente se mencionan los nombres. El apartado no incluye lo sugerido en la guía, como equipo de investigación y selección y capacitación del personal de campo, supervisión de la recolección de información, obtención de los datos según tipos de variables, estrategias de control de calidad de la recolección de los datos o diseño y manejo de las bases de datos. Debido a que la información brindada es muy limitada, este apartado ocupa dos renglones y no tres páginas, como lo sugiere la guía.

\section{Estudiante 3}

\section{Primera entrega}

En esta primera entrega, se evidencia el planteamiento del problema, que incluye: magnitud del problema, hallazgos de investigaciones sobre este, problema de investigación, brecha del conocimiento y para qué se van a usar los resultados del estudio. En la magnitud del problema, el estudiante no especifica información respecto al ámbito local; en cuanto a la brecha del conocimiento, expone la necesidad de abordar ciertos temas relacionados con el problema, pero no hace explícito el vacío de conocimiento; no presenta la pregunta de investigación ni cómo va a responderla. El tema de la investigación y el lugar donde se llevará a cabo están claramente definidos dentro del texto.

\section{Segunda entrega}

En esta entrega, se evidencia un documento más estructurado, que contiene portada, título del proyecto, planteamiento del problema, pregunta de investigación y objetivos. En cuanto al tema de la investigación, el estudiante conserva la línea investigativa esbozada en la primera entrega, de tal manera que muestra la información necesaria para que el lector comprenda el tema. Respecto al planteamiento del problema, con relación a la primera entrega, la magnitud del problema en el ámbito local ya está referenciada; la brecha del conocimiento no se hace explícita aún y la pregunta de investigación ya está definida, pero no se integra dentro del planteamiento, sino que se ubica de manera aislada. En cuanto a los objetivos, el general no abarca o se relaciona completamente con la pregunta de investigación, que es uno de los indicadores de la guía de escritura; los específicos son coherentes 
con el objetivo general, pero se formulan cinco. El estudiante cumple con los requisitos de forma: utiliza verbos en infinitivo y son medibles.

\section{Tercera entrega}

En esta última entrega, se evidencia un documento más completo, que incluye, además del planteamiento y los objetivos, la metodología del proyecto. El planteamiento del problema está mejor estructurado y la pregunta de investigación ya se encuentra integrada dentro de este; el objetivo general se relaciona de manera más coherente con la pregunta de investigación, a diferencia de la segunda versión. En cuanto a la metodología, se encuentran el tipo y el área de estudio, la población y la muestra, y las variables; en cuanto al tipo de estudio, aunque se presenta el panorama general, no se expone por qué se eligió ese tipo, los retos que supone y cómo se afrontarán, aspectos especificados en la guía de escritura. En el área de estudio, es claro el lugar en el que se va a desarrollar la investigación y la importancia de hacerla allí; además, el apartado cumple con la extensión de entre una y dos páginas requeridas. Respecto a la población y muestra, es muy general la descripción de las características de la población y no se evidencia la justificación de estudiar dicha muestra, el tamaño no es muy claro y no se explicitan los criterios de inclusión y exclusión. Por otro lado, las variables de la investigación son claras y se encuentran definidas dentro del texto.

El análisis de las versiones presentadas por los estudiantes permite identificar que los tres lograron hacer ajustes significativos en los apartados de planteamiento del problema y objetivos. La delimitación precisa y la suficiente documentación y argumentación del problema de investigación son aspectos clave para la definición de los demás apartados del anteproyecto. En los casos analizados, se observó que los ajustes en el problema de investigación tuvieron influencia en las precisiones de los objetivos, de tal forma que los tres estudiantes lograron una alta coherencia entre los dos apartados.

Con respecto a la metodología, es pertinente mencionar que los tres estudiantes solo presentaron una versión del apartado, a diferencia del problema y de los objetivos, por lo cual recibieron comentarios de sus pares y de las docentes, pero no alcanzaron a hacer modificaciones del texto en el curso seleccionado para la investigación. Como parte del proceso de escritura que se orienta en la maestría analizada, el objetivo de las últimas observaciones que los estudiantes recibieron es que las pudiesen retomar en el siguiente semestre en el curso 'Proyecto de Investigación II', cuyo insumo principal de trabajo es el anteproyecto elaborado durante la asignatura analizada.

Ahora bien, los apoyos que permitieron que los estudiantes fuesen precisando las versiones de sus anteproyectos estuvieron relacionados con las estrategias de lectura y organización de consultas bibliográficas, la revisión entre pares y por parte de las docentes del curso para ofrecer sugerencias escritas, la posibilidad de reescritura, y la especificación, a través de guías de escritura, de los criterios con los que debía cumplir cada apartado.

Con respecto a este último apoyo, cabe aclarar que las guías contemplaron criterios relevantes para orientar tanto el desempeño de los estudiantes como las evaluaciones de las docentes; sin embargo, se observó que los aspectos relacionados con el apartado de metodología fueron los que menos se pudieron cumplir a cabalidad. De acuerdo con los estudiantes, algunos de los elementos exigidos en la guía para la metodología eran difíciles de definir por el momento de desarrollo del proyecto, lo que ameritaba, por ejemplo, discusiones con sus directores de tesis que no alcanzaban a tener en el semestre analizado. De ahí la importancia de que se asuma la escritura del proyecto como un proceso que, si bien inicia y se orienta en el curso observado, tiene la posibilidad de continuar en las asignaturas siguientes y de nutrirse con los aportes de otros participantes importantes en el proceso de investigación y escritura, como, por ejemplo, los directores.

Esta particularidad sobre las guías también muestra la necesidad de flexibilidad con respecto al uso de estos instrumentos, dado que, aunque en cada versión se esperaba algo específico y las pautas fueron cruciales para orientar la escritura, se presentaron otros factores que incidieron en que 
se pudiesen seguir o no a cabalidad. En el caso analizado, se observa que las condiciones relacionadas con el momento de desarrollo las investigaciones no permitieron precisar todos los aspectos que se solicitaban en la metodología, y que estas circunstancias se salían del control de los estudiantes. No obstante, esto no resta importancia a la estrategia de apoyo mediada por el instrumento de la guía; por el contrario, brinda elementos precisos que pueden ser retomados por los estudiantes en los seminarios de investigación posteriores al cursado.

\section{Valoración de apoyos recibidos}

Respecto a los apoyos pedagógicos recibidos en la asignatura, los estudiantes resaltaron como útil e importante el módulo específico de escritura, en el que se abordaron estrategias procedimentales relacionadas con el proceso de escritura, tales como selección, organización y síntesis de bibliografía; revisión de borradores para ajustar aspectos gramaticales y de estructura; y revisión conjunta de modelos de proyectos del área de la salud pública para identificar asuntos retóricos. Para ellos, los principales aportes de este módulo tuvieron que ver con estrategias para organizar ideas (matrices de búsqueda y síntesis de referentes), conectarlas, revisar ortografía y otras particularidades de forma, que generalmente no tienen presentes al escribir; mencionan que fue fundamental trabajar todo lo anterior en función de la revisión y reescritura de las distintas versiones de sus anteproyectos. También destacaron los aportes de la docente en cuanto a estrategias de lectura, por ejemplo, lectura en voz alta y discusión de modelos de proyectos ya publicados en su campo de conocimiento, que eran asignados o recomendados por docentes especialistas en este.

Llama la atención que los estudiantes destacan, además de lo anterior, la orientación de la docente del área de Salud Pública en asuntos temáticos y en la precisión tanto de la pertinencia de la investigación como de sus alcances: la experiencia de investigadora de la docente de la asignatura es un apoyo vital para plantear el anteproyecto con más claridades. (Estudiante 2)

yo creo que son muy válidos los aportes [de la docente de Salud Pública], yo no tengo ninguna experiencia previa de escritura ni de publicación ni proyecto de grado ni nada, entonces fue como arrancar desde cero en toda la experiencia de escritura. [...] En general bien, porque uno va puliendo mejor la parte de la escritura. (Estudiante 3)

Con relación a las estrategias de apoyo ofrecidas por las docentes y más usadas por los estudiantes en su proceso de construcción del anteproyecto, mencionaron las siguientes: matrices para ubicar la información más relevante de la bibliografía consultada, tablas para organizar la información, el uso de textos modelo y el tiempo destinado en la clase para escribir; este último recurso fue catalogado por uno de los estudiantes como generador de distracciones, por lo que no lograba "dedicar un tiempo de calidad" (Estudiante 2) a la escritura, que era una de sus expectativas para el proceso.

Es posible evidenciar que todo lo anterior se corresponde con expectativas iniciales de los estudiantes con respecto a los apoyos pedagógicos, tales como: realimentación constante, estrategias para un mejor proceso de redacción, aspectos de forma y contenido. Además, agregan que los apoyos externos, como las asesorías con directores de tesis y asesorías personalizadas con la docente del curso que orientaba asuntos específicos del área de la salud para precisar aspectos temáticos y del planteamiento, facilitaron el proceso y aportaron de manera positiva a la escritura.

\section{Factores que influyen en la escritura y emociones percibidas durante el proceso}

En relación con la experiencia de escritura, se identificaron como barreras la carga académica de las otras materias, el desempeño de otros roles no académicos y la falta de organización de tiempo. Respecto a los factores favorecedores, los estudiantes mencionaron el tiempo de escritura destinado en clase, considerado también un apoyo pedagógico 
importante, la motivación que generaba avanzar en el proceso, los apoyos pedagógicos y la lectura constante de información para fortalecer la escritura.

Finalmente, en cuanto a las emociones y sentimientos que se generaron a partir del proceso de escritura, los estudiantes mencionan: "vergüenza cuando no se siente buena calidad en la escritura" (estudiante 2), "rabia, ira y frustración" (estudiante 3 ), identificando los momentos de entrega o plazos como generadores de estas emociones, "Angustia, frustración, estrés sobre todo en marco teórico y en la parte metodológica por falta de tiempo y llega un punto en que no sabes qué más escribir" y "motivación cuando uno va avanzando y va tomando forma" (estudiante 1). De acuerdo con ello, se evidencian en su mayoría sentimientos negativos, como el estrés y la frustración. Cuando se realizó la pregunta en las entrevistas, la respuesta en común fue la mención de emociones negativas como las que priman al escribir.

\section{Discusión y conclusiones}

A partir de este estudio se puede concluir que los conocimientos previos acerca de escritura de documentos de investigación que tienen los estudiantes influyen en las expectativas y valoraciones acerca de los apoyos pedagógicos recibidos por parte de los docentes, ya que se relacionan con la cantidad de estrategias para la lectura y la escritura del estudiante. Así lo menciona también Ochoa (2009): "quien ha recibido una formación adecuada en la producción escrita, tiene un entrenamiento y estrategias metacognitivas que le permiten afrontar la tarea de una manera no solo más adecuada sino menos estresante" (p. 115). Con relación a esto, se logró identificar que los apoyos pedagógicos brindados en la asignatura, en su mayoría, satisficieron las expectativas de los estudiantes, por lo que estos fueron valorados positivamente por todos; lo anterior se vio reflejado en las versiones de escritura del anteproyecto de grado de los estudiantes, pues estas fueron cada vez más completas y con más elementos en los diferentes apartados.
A pesar de que algunos de los puntos en los textos no cumplieron lo que se pedía en la guía de escritura, especialmente en el apartado de metodología, los estudiantes lograron elaborar un insumo importante que fue retomado en el semestre siguiente, lo que demuestra que en la maestría analizada se propone un proceso de escritura por fases a los estudiantes, que puede contribuir a la importante visión de que este proceso no debe asumirse en un solo momento, menos tratándose de un texto con las características de complejidad que tiene la tesis de maestría. Igualmente, esta propuesta de escribir por fases facilita el avance en el proceso de escritura, lo cual se relaciona con lo planteado por Arnoux et al. (2004), Franco (2016) y Pereira y Di Stefano (2007), quienes resaltan que el producto final del texto debe ser el resultado de constantes reformulaciones, lo que permite que el estudiante avance sobre borradores y logre identificar dificultades y estrategias utilizadas para la escritura.

De igual manera, se logró identificar que para los estudiantes hay más factores de dificultad que facilitadores durante el proceso de escritura. Lo mismo ocurrió respecto a las emociones percibidas, que fueron en su mayoría negativas, derivadas de los múltiples factores que dificultan el proceso y al mismo tiempo estas emociones son consideradas parte de las dificultades para la escritura, lo cual se relaciona con las conclusiones de la investigación realizada por Vargas Franco (2016) en el contexto de posgrado en una universidad colombiana. Asimismo, Ochoa Sierra y Cueva Lobelle (2017) encontraron que la angustia, el estrés, el miedo, el sufrimiento y la inseguridad son las principales emociones asociadas al proceso de escritura.

Para finalizar, es importante resaltar que en el curso que se analizó en esta investigación hubo una orientación explícita de la escritura, no desde una mirada remedial ni en un curso descontextualizado en el que se trabajan asuntos genéricos, sino que el abordaje se hizo para apoyar a los estudiantes en la escritura de su anteproyecto y en la comprensión de cómo funciona el discurso de su área particular. De acuerdo con las respuestas de los estudiantes, los aportes recibidos de la docente especialista en 
el área de salud y de la docente experta en escritura en el mismo curso constituyeron una estrategia de acompañamiento significativa en la construcción de sus anteproyectos, asunto que resalta la importancia del trabajo colaborativo entre docentes de distintas áreas y que pone en evidencia que la escritura de textos relacionados con la investigación no depende exclusivamente del abordaje de temáticas de metodología, sino que la reflexión y el trabajo acompañado sobre la escritura misma es esencial.

\section{Referencias}

Arnoux, E., Borsinger, A., Carlino, P., Di Stefano, M., Pereira, C. y Silvestri, A. (2004). La intervención pedagógica en el proceso de escritura de tesis de posgrado. Revista de la Maestría en Salud Pública, 2(3), 1-16.

Carlino, P. (2002). Leer, escribir y aprender en la universidad como lo hacen en Australia y por qué. Investigaciones en Psicología, 7(2), 43-61.

Carlino, P. (2005). ¿Por qué no se completan las tesis en los postgrados? Obstáculos percibidos por maestrandos en curso y magistri exitosos. Educere, 9(30), 415-420.

Carlino, P. (2009). Prácticas y representaciones de la escritura en la universidad: Los casos de Australia, Canadá, Ee. uU. y Argentina. Cuaderno de Pedagogía Universitario, 6(12), 6-17.

Carlino, P. (2013). Alfabetización académica diez años después. Revista Mexicana de Investigación Educativa, 18(57), 355-381.

Castelló, M. (2009a). Aprender a escribir textos académicos: ¿copistas, escribas, compiladores o escritores? En J. I. Pozo y M. de P. Pérez, Psicología del aprendizaje universitario: La formación en competencias (pp. 120-133). Ediciones Morata.

Castelló, M. (2009b). El proceso de composición de textos académicos. En M. Castelló, Escribir y comunicarse en contextos científicos y académicos (pp. 47-81). Graó.

Castelló, M., Iñesta, A. y Monereo, C. (2009). Towards Self-regulated Academic Writing: An Exploratory Study with Graduate Students in a Situated Learning Environment. Electronic Journal of Research on Educational Psychology, 1107-1130. https://doi. org/10.25115/ejrep.v7i19.1336.
Chirinos, N. M. (2012). Estrategias metacognitivas aplicadas en la escritura y comprensión lectora en el desarrollo de los trabajos de grado. Zona próxima, 17, 142-153.

Chois, P. M. y Jaramillo, L. G. (2016). La investigación sobre la escritura en posgrado: Estado del arte. Lenguaje, 44(2). https://doi.org/10.25100/lenguaje. v44i2.4622

Chois-Lenis, P. M., Arenas-Hernández, K. A., AguilarArias, A. y Mosquera-Becerra, J. (2020). Apoyar la escritura del proyecto de tesis en salud. Magis, Revista Internacional de Investigación en Educación, 12(25), 39-58.

Espino Datsira, S. (2015). La enseñanza de estrategias de escritura y comunicación de textos científicos y académicos a estudiantes de posgrado. Revista Mexicana de Investigación Educativa, 20(66), 959-976.

Flower, L. y Hayes, J. (1996). Teoría de la redacción como proceso cognitivo. En M. E. Rodríguez (dir.), Textos en contexto (pp. 75-110). Asociación Internacional de Lectura: Lectura y Vida.

Franco, J. (2016). Experiencia de intervención educativa para mejorar la calidad de escritura académica de los estudiantes de posgrado. CPU-e, Revista de Investigación Educativa, 22, 151-175. https://doi. org/10.25009/cpue.v0i22.1945

Hernández Sampieri, R., Fernández Collado, C. y Baptista Lucio, P. (2010). Metodología de la investigación. McGraw-Hill Education.

Mostacero, R. (2014). La tutoría grupal y entre iguales en el contexto de los estudios de posgrado. LegendaNueva Etapa, 18(18), 67-97.

Ochoa, L. (2009). La lectura y la escritura en la tesis de maestría. Forma y Función, 22(2), 93-119.

Ochoa Sierra, L. y Cueva Lobelle, A. (2017). El bloqueo en el proceso de elaboración de una tesis de maestría: Angustias y desazones percibidas por sus protagonistas. Lenguaje, 45(1), 61-87.

Pereira, C. y Di Stefano, M. (2007). El taller de escritura en posgrado: Representaciones sociales e interacción entre pares. Revista Signos, 40(64), 405-430. https:// doi.org/10.4067/s0718-09342007000200007

Torres, M. y Guadalupe, G. (2012). La escritura de tesis de posgrado en el área de investigación educativa. El acompañamiento, una pieza clave. CPU-e, Revista de Investigación Educativa, 15, 69-86. https://doi. org/10.25009/cpue.v0i15.21 
Universidad Pedagógica Nacional

Facultad de Humanidades

Uribe, G. y Camargo, Z. (2011). Prácticas de lectura y escritura académicas en la universidad colombiana. Revista Internacional de Investigación en Educación, 3(6), 317-341.

Vargas Franco, A. (2016). La escritura académica en el posgrado: la perspectiva del estudiante. Un estudio de caso. Revista de Docencia Universitaria, 97-129. https://doi.org/10.4995/redu.2016.5807

Zanotto, M., Monereo, C. y Castelló, M. (2011). Estrategias de lectura y producción de textos académicos: Leer para evaluar un texto científico. Perfiles Educativos, 14(1), 10-29. https://doi.org/10.22201/ iisue.24486167e.2011.133.27901 